\title{
Genetic horoscopes: is it all in the genes? Points for regulatory control of direct-to-consumer genetic testing
}

\author{
Christine Patch ${ }^{*, 1,2}$, Jorge Sequeiros ${ }^{1,3}$ and Martina C Cornel ${ }^{1,4}$
}

${ }^{1}$ Public and Professional Policy Committee of the European Society of Human Genetics, Vienna, Austria; ${ }^{2}$ Department of Clinical Genetics, NIHR Biomedical Research Centre, Guys and St Thomas' NHS Foundation Trust, Guys Hospital, London, UK; ${ }^{3}$ IBMC - Instituto de Biologia Molecular e Celular, and ICBAS, University of Porto, Porto, Portugal;

${ }^{4}$ Community Genetics, Department of Clinical Genetics/EMGO Institute, VU University Medical Center, Amsterdam, The Netherlands

The development of tests for genetic susceptibility to common complex diseases has raised concerns. These concerns relate to evaluation of the scientific and clinical validity and utility of the tests, quality assurance of laboratories and testing services, advice and protection for the consumer and the appropriate regulatory and policy response. How these concerns are interpreted and addressed is an ongoing debate. If the possibility of using the discoveries from genomic science to improve health is to be realised without losing public confidence, then improvements in the evaluation and mechanisms for control of supply of tests may be as important as the science itself.

European Journal of Human Genetics (2009) 17, 857-859; doi:10.1038/ejhg.2008.246; published online 4 March 2009

Keywords: direct-to-consumer; genetic tests; policy; clinical validity; utility; regulation

Genome-wide association studies often identify sets of genetic variants with small effects. But, much more work is then needed to validate those findings and establish their clinical relevance. Nevertheless, this has not stopped the rapid development of an emerging market of commercial genetic testing services, on the basis of these associations alone. Often, these are marketed directly to the consumer, as a way of providing health-related information or lifestyle choices. Commentators have raised concerns about (1) the scientific and clinical validity of these tests and their clinical utility, (2) the quality of the testing services and (3) the appropriate regulatory and policy response. ${ }^{1}$ The European Society of Human Genetics thus

*Correspondence: Dr C Patch, Department of Clinical Genetics, NIHR Biomedical Research Centre, Guys and St Thomas NHS Foundation Trust, 7th Floor Borough Wing, Guys Hospital, Great Maze Pond Road, London SE1 9RT, UK.

Tel: + 44207188 1364; Fax: + 44207188 1369;

E-mail: christine.patch@gstt.nhs.uk

Received 9 July 2008; revised 10 November 2008; accepted 20 November 2008; published online 4 March 2009 needs to consider these developments and challenges, as well as the issue of guiding principles that may promote the effective translation of research and innovation into responsible services in the area of genetic testing.

A recent critical appraisal proposed there is insufficient evidence to conclude that genomic profiling is useful in measuring individual genetic risks for common diseases, or in developing personalized diet and lifestyle recommendations for disease prevention. ${ }^{2}$ Another analysis suggested that this genetic astrology could be regarded as producing no more than entertaining horoscopes; there is, however, a potential for harm and the need to consider mechanisms to ensure that these tests are evaluated and used appropriately. $^{3}$

In 2006, the US Government Accountability Office initiated an investigation, in which a number of companies, randomly selected, were provided with samples for testing. They concluded that the information reported after analysis was medically unproven and ambiguous. ${ }^{4}$ After an investigation by the Secretaries Advisory Committee on Genetics Health and Society, the call was made to issue 
a consumer alert. $^{5}$ The Federal Trade Commission, together with the Food and Drug Administration (FDA) and the Centre for Disease Control, subsequently did so with the statement 'At home genetic tests: a healthy dose of scepticism may be the best prescription' ${ }^{6}$ In 2008 , the American College of Medical Genetics issued a policy statement on direct-to-consumer genetic testing, which sets out a series of recommendations, including that a test should be ordered and interpreted only by a knowledgeable health professional. ${ }^{7}$ More recently, the California Department of Public Health issued a 'cease and desist letter' to 13 genetic testing laboratories, following a similar action by the New York State Department of Health in April.

In the United States, oversight of genetic tests is the responsibility of the FDA. Tests that fall under their jurisdiction have to go through a process of extensive premarket review, before being allowed to market. There is an exemption, however, for those tests that are based in the laboratory that created them - so called 'home-brew tests' may be regulated through the Clinical Laboratory Improvement Act, which requires only evidence of analytical validity. In the case of a genetic test, the assay may be analytically valid, if it accurately identifies a particular polymorphism or mutation; however, if that genetic variant has nothing to do with the disease(s) of interest, then the test has no clinical validity. ${ }^{8}$ Examples can be found in the paper by Janssens et al. ${ }^{2}$ Only two out of five genes included in 'osteogenomic profiles' were significantly associated with some disease, but none were associated with bone disease.

Furthermore, clinical utility is often unknown or unlikely. Several companies advertise a test, as being aware of an increased risk might increase a consumer's motivation for preventive behaviours. As few effective behavioural interventions are available, however, an unfavourable test result will rarely lead to a longer and healthier life. It may be that an individual will attach more importance to knowing their risk, than to the availability of interventions. However, even if analytical and clinical validity are established, claims made relating to clinical utility demand a test with adequate predictive value as well as the availability of an effective preventative strategy.

In contrast to the United States, Canada and Australia, where regulators have placed genetic tests into a higher risk category requiring greater oversight, the majority of tests within Europe are classified as low risk, meaning that claims are not reviewed before tests are marketed and test marketing is based on a system of self certification. ${ }^{9}$

The UK Human Genetics Commission (HGC), in its 2003 report Genes Direct and the 2008 follow up report 'More Genes Direct', has made a number of recommendations in relation to the oversight of genetic tests provided direct to the public. ${ }^{10,11}$ The HGC recommended stricter controls on genetic testing, but believed that there should not be statutory prohibition of some or all direct genetic tests and that consideration should be given to the statement that certain genetic tests should be provided only by an appropriately qualified health professional.

These recommendations relating to oversight are clustered around three areas: premarket review, quality assurance and advice and advertising. The recommendations arose out of a meeting of expert stakeholders and include calls for (1) revisiting of the risk classification under the European IVD Directive, ${ }^{12}$ allowing for greater scrutiny of the evidence at the premarket review stage, (2) the development of a code of practice for test suppliers and (3) transparency in relation to the evidence or lack of evidence for the claims made by test suppliers and manufacturers. A follow-up meeting has recently been held involving members of regulators, policy makers and commercial test suppliers from Europe and the USA, which supported the development of a code of practice. The HGC is now leading on the production of a 'Common Framework of Principles' for the provision of direct genetic tests. ${ }^{13}$ The intention is that the principles will apply across all jurisdictions and build on existing international guidelines and protocols.

The Organisation for Economic Cooperation and Development has also recently produced guidelines for the quality assurance of molecular genetic testing, which recommend that (1) this should be delivered within the framework of health care, (2) this should be practised under a quality assurance framework, (3) this should be in compliance with applicable legal, ethical and professional standards, (4) that advertising, promotional and technical claims for molecular genetic tests and devices should accurately describe the characteristics and limitations of the tests offered, (5) that test results should be reported back to the referring health-care professional and (6) that appropriate pre- and post-test counselling should be available. ${ }^{14}$

On the 7th of May, the Committee of Ministers of the Council of Europe adopted a new Additional Protocol to the Convention on Human Rights and Biomedicine concerning Genetic Testing for Health Purposes. ${ }^{15}$ In this protocol, recommendations are made that (1) genetic tests meet generally accepted criteria of scientific validity and clinical validity, (2) clinical utility should be an essential criterion for a test to be offered, (3) a quality assurance programme should be implemented, (4) adequate previous information is provided whenever a test is considered, (5) appropriate genetic counselling should be available in the case of predictive tests and (6) persons providing genetic services have appropriate qualifications, to enable them to perform their role in accordance with professional obligations and standards.

Although most debate has concerned itself with the Webbased companies advertising and offering a laboratory assay, without necessarily involving a medical intermediary, 
genetic tests on the basis of discovery from genome-wide association studies are increasingly being offered through private and public medical services, which often offer them directly to the public. Concerns relating to clinical validity and quality assurance apply as much to this latter model of service provision as they do to the former.

Some companies also store DNA so that customers can use their genetic information again at a later stage. There should be discussion of the privacy and consent issues in relation to the storage and analysis of samples. Two particular concerns may be how to guarantee that the sample belongs to the sender and how samples belonging to minors are handled, given that there are concerns expressed relating to the appropriate consent for genetic tests for adult onset conditions in asymptomatic minors where there is no clinical benefit at present. $^{16}$

Translational medicine demands more than an advertisement and an information leaflet. ${ }^{17}$ As the above recommendations indicate, there are various points of control in relation to the mechanisms for oversight of genetic tests provided directly to the public. (1) Statutory control should require transparency of evidence of the claims that are made by manufacturers or tests providers, before the test comes to market. (2) Professional bodies and codes of practice should ensure that those offering, providing and interpreting genetic tests are working within an appropriate framework, and only offer tests for which there is evidence of clinical utility. (3) Routine use of tests should be reimbursed only after they have been evaluated effectively. (4) Advertising and promotional materials should not make misleading claims, and appropriate protection for the consumer should be in place. (5) Regulation on storage and privacy protection should follow international guidance for biobanks and genetic information, including guidance on samples from minors. (6) Commercial companies should not analyse samples of minors for genetic conditions, unless the tests are ordered by health professionals.

How this is interpreted and put into place, at the policy level, is an ongoing debate and may differ from country to country. Harmonization of practices and regulations and international cooperation among regulatory agencies need to be promoted, particularly given the increasing flow of samples across the borders. Also, genetic literacy of health professionals, the media and the public will be of the utmost importance.

If the promise of genomics to improve health is to be realized, without causing harm or loss of public trust, then improvements in the evaluation and control of supply of tests may be as important as the science itself.

\section{References}

1 Hogarth S, Javitt G, Melzer D: The current landscape for directto-consumer genetic testing: legal, ethical, and policy issues. Annu Rev Genomics Hum Genet 2008; 9: 161-182.

2 Janssens AC, Gwinn M, Bradley LA, Oostra BA, van Duijn CM, Khoury MJ: A critical appraisal of the scientific basis of commercial genomic profiles used to assess health risks and personalize health interventions. Am J Hum Genet 2008; 82: 593-599.

3 Melzer D, Hogarth S, Liddell K, Ling T, Sanderson S, Zimmern RL: Genetic tests for common diseases: new insights, old concerns. BMJ 2008; 336: 590-593.

4 United States Government Accountability Office (GAO): Nutrigenetic Testing: Tests Purchased from Four Web Sites Mislead Consumers. Jul 2006. Available from: URL: http://frwebgate.access.gpo.gov/cgi-bin/ getdoc.cgi?dbname $=$ gao $\&$ docid $=$ f:d06977t.pdf.

5 Secretary's Advisory Committee on Genetics, Health, and Society (SACGHS): US system of oversight of genetic testing: a response to the charge of the Secretary of Health and Human Services (report). Apr 2008. Available from: URL: http://oba.od.nih.gov/ oba/SACGHS/reports/SACGHS_oversight_report.pdf.

6 Federal Trade Commission (FTC): FTC facts for consumers: at-home genetic tests - a healthy dose of skepticism may be the best prescription. Jul 2006. Available from: URL: http:// www.ftc.gov/bcp/edu/pubs/consumer/health/hea02.shtm.

7 American College of Medical Genetics (ACMG): What the public needs to know about direct-to-consumer genetic tests. Policy Statement, Apr 2008. Available from: URL: http://www.acmg.net/ AM/Template.cfm?Section $=$ Policy_Statements\&Template $=/ \mathrm{CM} /$ ContentDisplay.cfm\&ContentID $=2975$.

8 Schmidt C: Regulators weigh risks of consumer genetic tests. Nat Biotech 2008; 26: 145-146.

9 Hogarth S, Melzer D: The IVD directive and genetic testing problems and proposals. A briefing presented to the 20th Meeting of Competent Authorities. Lisbon, July 2007. Available from: URL: http://www.eurogentest.org/web/db/news/167/index.xhtml.

10 Human Genetics Commission: Genes direct: ensuring the effective oversight of genetic tests supplied directly to the public, Mar 2003. Available from: URL: http://www.hgc.gov.uk/UploadDocs/DocPub/ Document/genesdirect_full.pdf.

11 Human Genetics Commission: More genes direct: a report on developments in the availability, marketing and regulation of genetic tests supplied directly to the public, Dec 2007. Available from: URL: http://www.hgc.gov.uk/UploadDocs/DocPub/Document/ More\%20Genes\%20Direct\%20-\%20final.pdf.

12 Directive 98/79/EC of the European Parliament and of the Council of 27 October 1998 on in vitro medical devices. Available from: URL: http://ec.europa.eu/enterprise/medical devices/guide-stds-directives/cons_vers_98_79_ec.pdf.

13 Human Genetics Commission Common Framework of Principles for Direct Genetic Tests http://www.hgc.gov.uk/Client/ Content.asp?ContentId $=816$.

14 OECD/OCDE. OECD guidelines for quality assurance in molecular genetic testing. 2007. Available from: URL: http://www. oecd.org/dataoecd/43/6/38839788.pdf.

15 Council of Europe: additional protocol to the convention on human rights and biomedicine, concerning genetic testing for health purposes. May 2008 Available from: URL: https://wcd.coe. int/ViewDoc.jsp?id $=1287907 \&$ Site $=$ DC\&BackColorInternet $=$ F5CA75\&BackColorIntranet $=$ F5CA75 $\&$ BackColorLogged $=$ A9BACE.

16 Borry $\mathrm{P}$ et al: Genetic testing in asymptomatic minors. 2008 Available from: URL: http://www.eshg.org/documents/ TestingInMinorsBackground.pdf.

17 van Ommen GJB, Cornel MC: Recreational genomics? Dreams and fears on genetic susceptibility screening. Eur J Hum Genet 2008; 16: 403-404. 